\title{
MicroRNA-187 promotes growth and metastasis of gastric cancer by inhibiting FOXA2
}

\author{
CONG LI ${ }^{1}$, SUMEI LU ${ }^{2}$ and YUBIN SHI \\ Departments of ${ }^{1}$ Emergency Internal Medicine, and ${ }^{2}$ Internal Neurology, \\ Tongren Hospital, Shanghai Jiao Tong University School of Medicine, Shanghai 200025, P.R. China
}

Received June 21, 2016; Accepted November 14, 2016

DOI: $10.3892 /$ or.2017.5370

\begin{abstract}
MicroRNAs (miRNAs) play an active role in the pathogenesis of gastric cancer. The expression and biological function for miR-187 in gastric cancer remains unknow. In the present study, we demonstrated that miR-187 expression was increased in gastric cancer (GC) tissues and cells. Increased expression level of miR-187 was associated with adverse clinical features including tumor size, lymph metastasis and TNM stage, and decreased overall survival and disease-free survival of GC patients. Functionally, overexpression miR-187 could promote while inhibition of miR-187 could suppress, the proliferation, migration and invasion of GC cells in vitro. In vivo experiments showed that overexpression of miR-187 promoted the growth and lung metastasis of SGC-7901 cells in nude mice. Mechanically, we confirmed that FOXA2 was the downstream target of miR-187 in GC cells using luciferase assay, qRT-PCR and western blot analysis. Moreover, overexpression of FOXA2 abrogated the promoting effects of miR-187 overexpression on SGC-7901 cell proliferation, migration and invasion, while inhibition of FOXA2 reversed the inhibitory effects of miR-187 downregulation on these biological functions of AGS cells, suggesting that FOXA2 was a functional mediator of miR-187 in GC. Therefore, this study indicates that miR-187 is potentially a biomarker and treatment target for GC patients.
\end{abstract}

\section{Introduction}

Gastric cancer (GC), with approximately 1 million new diagnosed patients every year (1), is the fourth most common human cancer and ranks second as leading cause of cancerrelated mortality worldwide (2). For patients in advanced stage of $\mathrm{GC}$, the prognosis is very poor, with a 5-year overall

Correspondence to: Dr Yubin Shi, Department of Emergency Internal Medicine, Tongren Hospital, Shanghai Jiao Tong University School of Medicine, 1111 Xianxia Road, Shanghai 200025, P.R. China

E-mail: yubinshi16621@163.com

Key words: miR-187, gastric cancer, FOXA2, tumor growth, metastasis survival less than 30\% (3). Malignant growth and systemic metastasis are the major reasons for the unsatisfactory survival of GC patients in advanced stage. Therefore, clarifying the molecular mechanisms for the development and progression of GC will be critical for identifying novel therapeutic targets for GC patients.

MicroRNAs (miRNAs), a class of short non-coding RNA sequences, play versatile roles in cellular processes including cell proliferation, apoptosis and movement (4). In addition, miRNAs have been confirmed to be aberrantly expressed and play fundamental roles in tumorigenesis and progression of human cancers (5) including GC (6). Several miRNAs were suggested as novel biomarkers and therapeutic targets in GC, including let7 (7), miR-196a (8), miR-101 (9) and miR-130b (10).

Recently, miR-187, a novel cancer-related microRNA, has been reported to play important roles in non-small cell lung (11), colorectal (12), prostate (13) and breast cancer (14). In non-small cell lung cancer, miR-187 was found to promote the cancer development by inhibiting Bcl-6 (11). Besides, the study of breast cancer showed that miR-187 is a prognostic marker and could potentiate the invasive ability of cancer cells (14). However, miR-187 was found to be downregulated in prostate cancer and was correlated with adverse clinicopathological features of patients (13). In addition, in colorectal cancer, miR-187 was found to be the downstream target of TGF $\beta$ pathway and could suppress Smad-mediated epithelial-mesenchymal transition in colorectal cancer cells (12). Therefore, the expression and function of miR-187 vary between different human types of cancers. However, the expression and function of miR-187 in GC remain uninvestigated.

The present study found that miR-187 was significantly upregulated in GC tissues. Increased expression of miR-187 was associated with poor clinicopathological features and worse survival of GC patients. Functionally, miR-187 enhanced proliferation, migration and invasion of GC cells both in vitro and in vivo. Moreover, we found that FOXA2 was a direct downstream target of miR-187 and mediated the biological functions of miR-187 in GC cells.

\section{Materials and methods}

Cell cultures. Gastric cancer cell lines SGC-7901, MKN-45, MGC-803 and normal gastric epithelial GES-1 cells were 
Table I. Clinical association analysis of miR-187 expression in gastric cancer.

No. of patients

\begin{tabular}{|c|c|c|c|c|}
\hline \multirow{2}{*}{ Clinicopathological features } & \multirow[b]{2}{*}{ Total no. of patients $(n=100)$} & & \multirow[b]{2}{*}{ P-value } \\
\hline & & miR-187 $7^{\text {low }}$ group & miR-187 $7^{\text {high }}$ group & \\
\hline \multicolumn{5}{|l|}{ Age (years) } \\
\hline$<65$ & 54 & 25 & 29 & \multirow{2}{*}{0.547} \\
\hline$\geq 65$ & 46 & 25 & 21 & \\
\hline \multicolumn{5}{|l|}{ Gender } \\
\hline Male & 68 & 31 & 37 & \multirow[t]{2}{*}{0.284} \\
\hline Female & 32 & 19 & 13 & \\
\hline \multicolumn{5}{|l|}{ Size $(\mathrm{cm})$} \\
\hline$<5$ & 33 & 24 & 9 & \multirow[t]{2}{*}{$<0.001$} \\
\hline$\geq 5$ & 47 & 6 & 41 & \\
\hline \multicolumn{5}{|l|}{ Tumor depth } \\
\hline $\mathrm{T} 1$ & 26 & 11 & 15 & \multirow[t]{2}{*}{0.494} \\
\hline $\mathrm{T} 2-\mathrm{T} 4$ & 74 & 39 & 35 & \\
\hline \multicolumn{5}{|l|}{ Lymph node metastasis } \\
\hline Absent & 34 & 27 & 7 & \multirow[t]{2}{*}{$<0.001$} \\
\hline Present & 66 & 23 & 43 & \\
\hline \multicolumn{5}{|l|}{ Venous infiltration } \\
\hline Absent & 70 & 39 & 31 & \multirow[t]{2}{*}{0.126} \\
\hline Present & 30 & 11 & 19 & \\
\hline \multicolumn{5}{|l|}{ TNM stage } \\
\hline I, II & 56 & 41 & 15 & \multirow[t]{2}{*}{$<0.001$} \\
\hline III, IV & 44 & 9 & 35 & \\
\hline
\end{tabular}

purchased from the American Type Culture Collection (ATCC; Rockville, MD, USA) and the Cell Bank of Chinese Academy of Sciences (Shanghai, China). All cells were cultured in RPMI-1640 medium (Life Technologies, Inc., Gaithersburg, MD, USA) supplemented with $10 \%$ fetal bovine serum (FBS; Gibco, Grand Island, NY, USA), penicillin (100 U/ml) and streptomycin $(100 \mathrm{mg} / \mathrm{ml})$. All cell cultures were kept at $37^{\circ} \mathrm{C}$ in a humidified incubator with $5 \% \mathrm{CO}_{2}$.

Clinical tissues. The approval to conduct the experiments involving human tissue samples was obtained from the Institutional Research Ethics Committee of Tongren Hospital, Shanghai Jiao Tong University School of Medicine. One hundred pairs of gastric cancer tissues and adjacent non-tumor tissues were collected from Tongren Hospital, Shanghai Jiao Tong University School of Medicine. All these clinical tissues were stored at $-80^{\circ} \mathrm{C}$ before the RNA extraction. The informed consents were obtained from all enrolled patients in this study. Demographic and clinicopathological information of the included patients are presented in Table I.

Cell transfection. The mimic and the inhibitor of miR-187 were obtained from Ruibobio, Guangzhou, China. FOXA2 siRNA and FOXA2 expression vector was purchased from SigmaAldrich (St. Louis, MO, USA). The day before the transfection, GC cells were seeded in 6-well plates. Then, miR-187 mimic or inhibitor/FOXA2 siRNA or plasmid were transfected into
GC cells with Lipofectamine 2000 (Invitrogen, Carlsbad, CA, USA) following the manusfacturer's protocol.

Real-time quantitative reverse transcription-PCR ( $q R T-P C R)$. Total RNA from GC tissues and cells were extracted with TRIzol reagent (Invitrogen). Reverse transcription reactions and real-time PCR were performed with Transcriptor First Strand cDNA Synthesis kit (Roche, Indianapolis, IN, USA) and SYBR-Green PCR Master Mix (Applied Biosystems, Foster City, CA, USA). Primers for miR-187 and U6 were purchased from GeneCopoeia (Guangzhou, China). U6 was used as the control gene for the relative expression level of miR-187.

Western blot analysis. Western blot analysis was performed following standard protocols. Generally, cellular protein was extracted with the RIPA lysis buffer, and protein concentration was measured using the BCA kit (Pierce, Rockford, IL, USA). A total of 20-40 $\mu \mathrm{g}$ cellular proteins were separated by SDS-PAGE and transferred to PVDF membrane. The following primary antibodies including FOXA2 (1:1,000; Cell Signaling Technology, Danvers, MA, USA) and GAPDH (1:2,000; Santa Cruz Biotechnology, Santa Cruz, CA, USA) were incubated with the membrane overnight at $4^{\circ} \mathrm{C}$. The blots were then incubated with secondary antibodies (1:3,000; Santa Cruz Biotechnology) at room temperature for $2 \mathrm{~h}$. The signals were visualized with ECL reagents (Amersham Biosciences Corp., Piscataway, NJ, USA). 

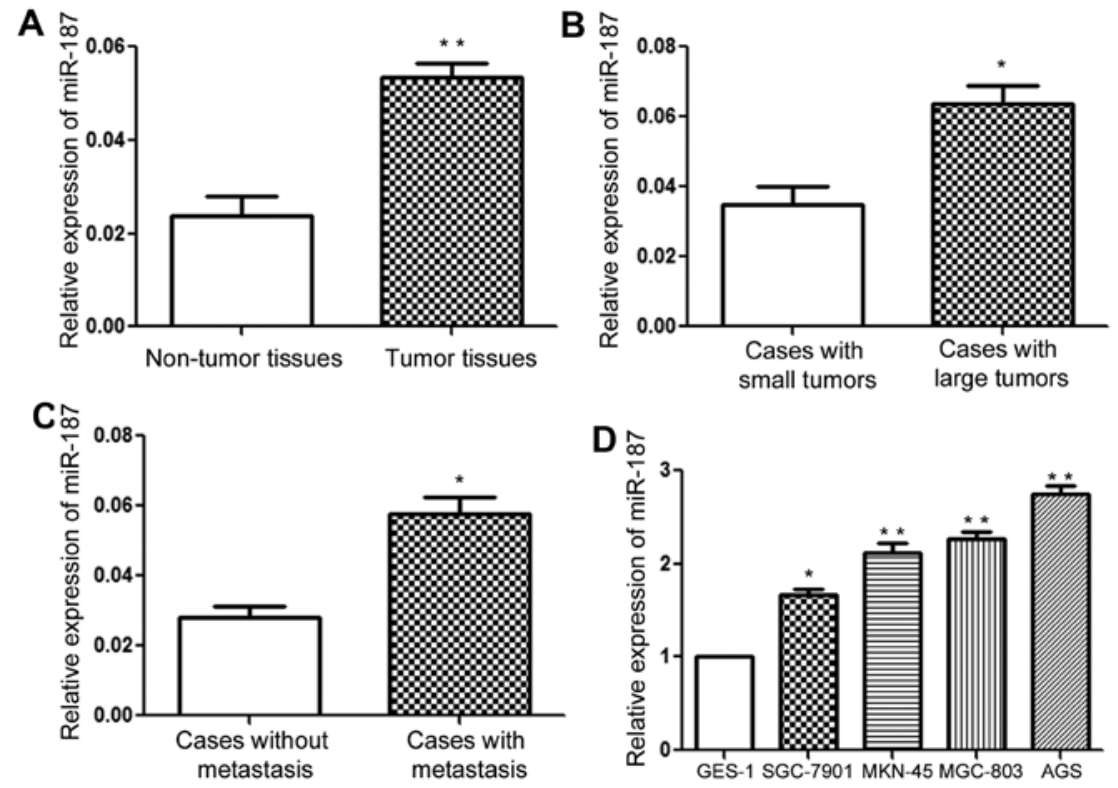

Figure 1. The expression status of miR-187 in gastric cancer. (A) qRT-PCR results for the expression of miR-187 in gastric cancer tissues and matched adjacent normal tissues. (B) Relative expression level of miR-187 in patients with small tumors and those with large tumors. (C) Relative expression level of miR-187 in patients with metastasis and those without metastasis. (D) Relative expression level of miR-187 in gastric cancer cell lines and the GES-1 cells. "P<0.05, ${ }^{* *} \mathrm{P}<0.01$.

MTT. For MTT assay, 5,000 GC cells transfected with miR-187 mimic or inhibitor were plated into 96-well plates. At 24, 28 and $72 \mathrm{~h}$ after cell seeding, the cells were stained with MTT (Sigma-Aldrich) for $4 \mathrm{~h}$ at $37^{\circ} \mathrm{C}$, and then the absorbance at $490 \mathrm{~nm}$ was examined.

Wound healing assay. GC cells were cultured to confluence in 6-well plates. A $100-\mu 1$ pipette tip was used to make the wounds on the confluent cells. The width of wounds was measured at 0 and $24 \mathrm{~h}$ after scratching and then the percentage of wound healing was calculated.

Transwell assays. The invasive ability of GC cells was evaluated by Transwell assays. Generally, upper chamber of Transwell device was coated with $80 \mu \mathrm{l}$ mixture of RPMI-1640 and Matrigel with a ratio of 1:8. Then, GC cells suspended in serum-free RPMI-1640 medium were plated in the upper chamber while $700 \mu \mathrm{l}$ serum-containing medium RPMI-1640 were placed in the lower chamber. Twenty-four hours later, GC cells invaded into the lower surface were stained with crystal violet. Cell number for the invaded cells was counted under a microscope.

Luciferase assay. Wild-type 3'-UTR sequence of FOXA2 containing the miR-187 predicted target site or the mutated sequence within the predicted target sites was cloned into the pGL3 control vector (Promega, Madison, WI, USA), designated as the wild-type FOXA2-3'-UTR or mutant FOXA2-3'-UTR, respectively. Then, GC cells seeded into 12-well plates were maintained in Opti-MEM reduced serum media (Life Technologies) the day before the transfection, and were cotransfected with wild-type or mutant 3'-UTR of FOXA2 along with miR-187 mimic or inhibitor. Forty-eight hours after the transfection, luciferase activity was measured by a single luciferase reporter assay (Promega).
In vivo tumor growth and metastasis assay. All animal experiments were approved by the Animal Care Committee of Tongren Hospital, Shanghai Jiao Tong University School of Medicine. For tumor growth studies, nude mice were injected subcutaneously with $1 \times 10^{6}$ cancer cells transfected with control vector or miR-187 mimic. Tumor sizes were measured every three days after cancer cell injection. Three weeks later, tumors were removed and the volumes were measured. For metastasis assay, GC cells were injected through tail vein into nude mice. Eight weeks later, the lungs of nude mice were subjected to H\&E staining for potential lung metastasis of GC cells.

Statistical analysis. All quantatively data are presented as mean \pm standard error of the mean (SEM). Statistical analysis including Student's t-test, Chi-square, correlation analysis and Kaplan-Meier analysis was performed with SPSS software (SPSS, Inc., Chicago, IL, USA) and GraphPad software. $\mathrm{P}<0.05$ was considered statistically significant.

\section{Results}

miR-187 expression is increased in GC tissues and cells. We performed qRT-PCR to determine the expression status of miR-187 in GC tissues. Compared with adjacent normal tissues, the expression of miR-187 in GC tissues was increased significantly (Fig. 1A; $\mathrm{P}<0.01$ ). In addition, compared to those of small size, tumors of large size had significantly increased level of miR-187 (Fig. 1B; P<0.05). Furthermore, compared to those without metastasis, the expression level of miR-187 in patients with metastasis was significantly elevated (Fig. 1C; $\mathrm{P}<0.05)$. Moreover, we examined the expression of miR-187 in GC cells and the normal gastric epithelial GES-1 cells. Compared to GES-1 cells, miR-187 in GC cells lines was significantly increased (Fig. 1D; $\mathrm{P}<0.05$ ). Besides, among the 

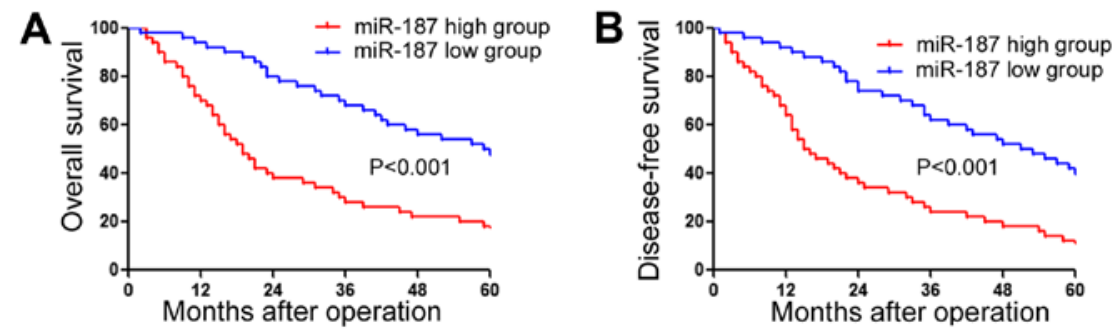

Figure 2. The prognostic value of miR-187 in gastric cancer. (A) The overall survival was compared between patients with high level of miR-187 and those with low miR-187 level. (B) The disease-free survival was compared between patients with high level of miR-187 and those with low miR-187 level.
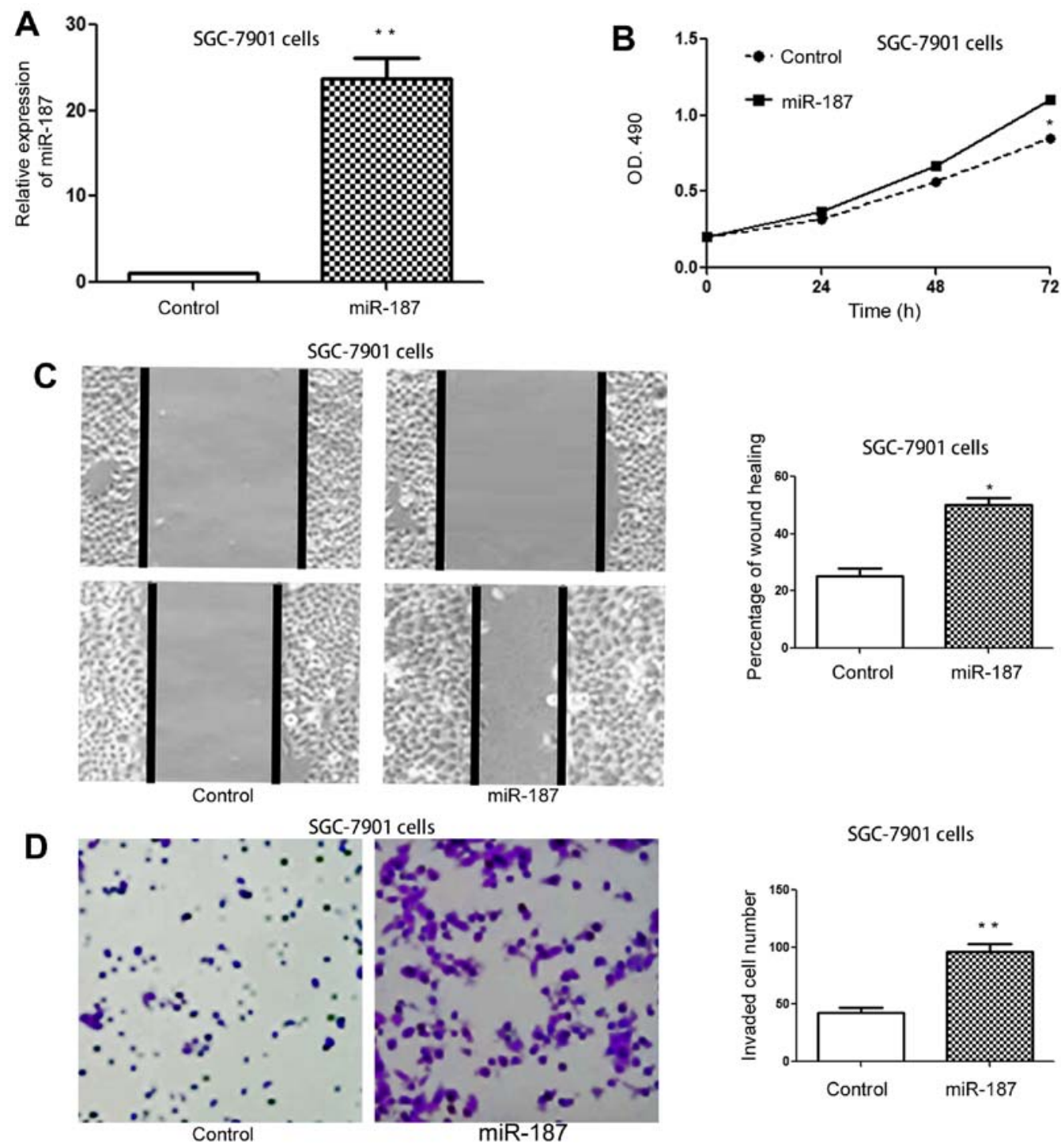

Figure 3. Overexpression of miR-187 promotes proliferation, migration and invasion of SGC-7901 cells. (A) miR-187 mimic significantly increased the expression of miR-187 in SGC-7901 cells. (B) The proliferation of SGC-7901 cells was significantly enhanced after overexpression of miR-187, as determined by MTT assay. (C) The migration of SGC-7901 cells was significantly enhanced after overexpression of miR-187, as determined by wound healing assay. (D) The invasion of SGC-7901 cells was significantly enhanced after overexpression of miR-187, as determined by Transwell assay. ${ }^{*} \mathrm{P}<0.05,{ }^{* *} \mathrm{P}<0.01$.

four GC cell lines, miR-187 level was lowest in SGC-7901 cells while was highest in AGS cells (Fig. 1D), indicating that miR-187, which is elevated in GC tissues and cells, probably plays an oncogenic role in GC.

Increased miR-187 expression is associated with poor clinicopathological features and prognosis of GC patients. After confirming the increased expression of miR-187 in GC, we further determined whether miR-187 expression level was associated with clinical features and the survival of GC patients. Association analysis (Table II) showed that high expression level was associated with large tumor size $(\mathrm{P}<0.001)$, lymphatic metastasis $(\mathrm{P}<0.001)$ and advanced TNM stage $(\mathrm{P}<0.001)$. Furthermore, survival analysis showed the patients 
Table II. Multivariate Cox regression analysis of 5-year overall and disease-free survival of 80 gastric cancer patients.

\begin{tabular}{|c|c|c|c|c|c|c|}
\hline \multirow[b]{2}{*}{ Variables } & \multicolumn{3}{|c|}{ Overall survival } & \multicolumn{3}{|c|}{ Disease-free survival } \\
\hline & HR & $95 \% \mathrm{CI}$ & P-value & HR & $95 \% \mathrm{CI}$ & P-value \\
\hline Histology & 0.557 & $0.319-0.971$ & $0.039^{\mathrm{a}}$ & 0.686 & $0.390-1.205$ & 0.190 \\
\hline Size $(\mathrm{cm})$ & 2.344 & $1.482-3.706$ & $<0.001^{\mathrm{a}}$ & 1.355 & $0.825-2.225$ & 0.229 \\
\hline Stage & 3.188 & $1.964-5.175$ & $<0.001^{\mathrm{a}}$ & 2.660 & $1.588-4.457$ & $<0.001^{\mathrm{a}}$ \\
\hline miR-340 expression & 2.269 & $1.472-3.498$ & $0.001^{\mathrm{a}}$ & 1.604 & $1.011-2.543$ & $0.045^{\mathrm{a}}$ \\
\hline
\end{tabular}

HR, hazard ratio; CI, confidence interval. a Statistically significant.
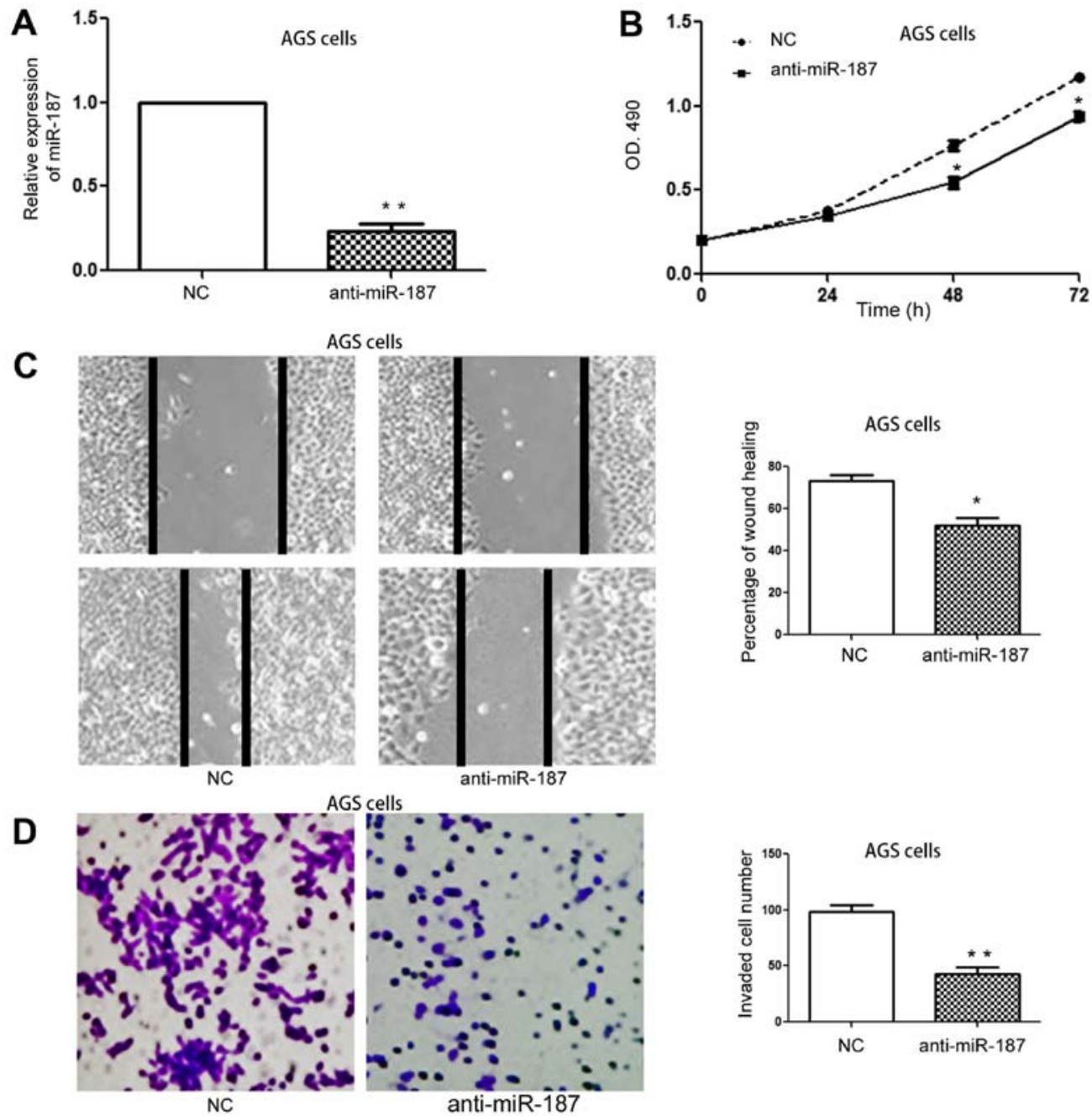

Figure 4. Inhibition of miR-187 decreases the proliferation, migration and invasion of AGS cells. (A) miR-187 inhibitor significantly decreased the expression of miR-187 in AGS cells. (B) The proliferation of AGS cells was significantly decreased after inhibition of miR-187, as determined by MTT assay. (C) The migration of AGS cells was significantly reduced after inhibition of miR-187, as determined by wound healing assay. (D) The invasion of AGS cells was significantly decreased after inhibition of miR-187, as determined by Transwell assay. ${ }^{*} \mathrm{P}<0.05,{ }^{* *} \mathrm{P}<0.01$.

with high level of miR-187 had significantly decreased overall survival (Fig. 2A; P<0.001) and disease-free survival (Fig. 2B; $\mathrm{P}<0.001)$. These data indicate that miR-187 is actively involved in the pathogenesis of GC, and can potentially serve as a novel biomarker for the prognosis of GC patients.

miR-187 promotes the proliferation, migration and invasion of GC cells. Aberrantly increased expression of miR-187 in
GC prompted us to explore the biological function of miR-187 in GC cells. Transfection of miR-187 mimic in SGC-7901 cells significantly increased miR-187 expression in SGC-7901 cells (Fig. 3A; P<0.01). Functionally, overexpression of miR-187 obviously increased cell proliferation of SGC-7901 cells (Fig. 3B; P<0.05) as suggested by MTT assay. Moreover, the wound healing assay and Transwell assay showed that the migration and invasion of SGC-7901 was obviously increased 

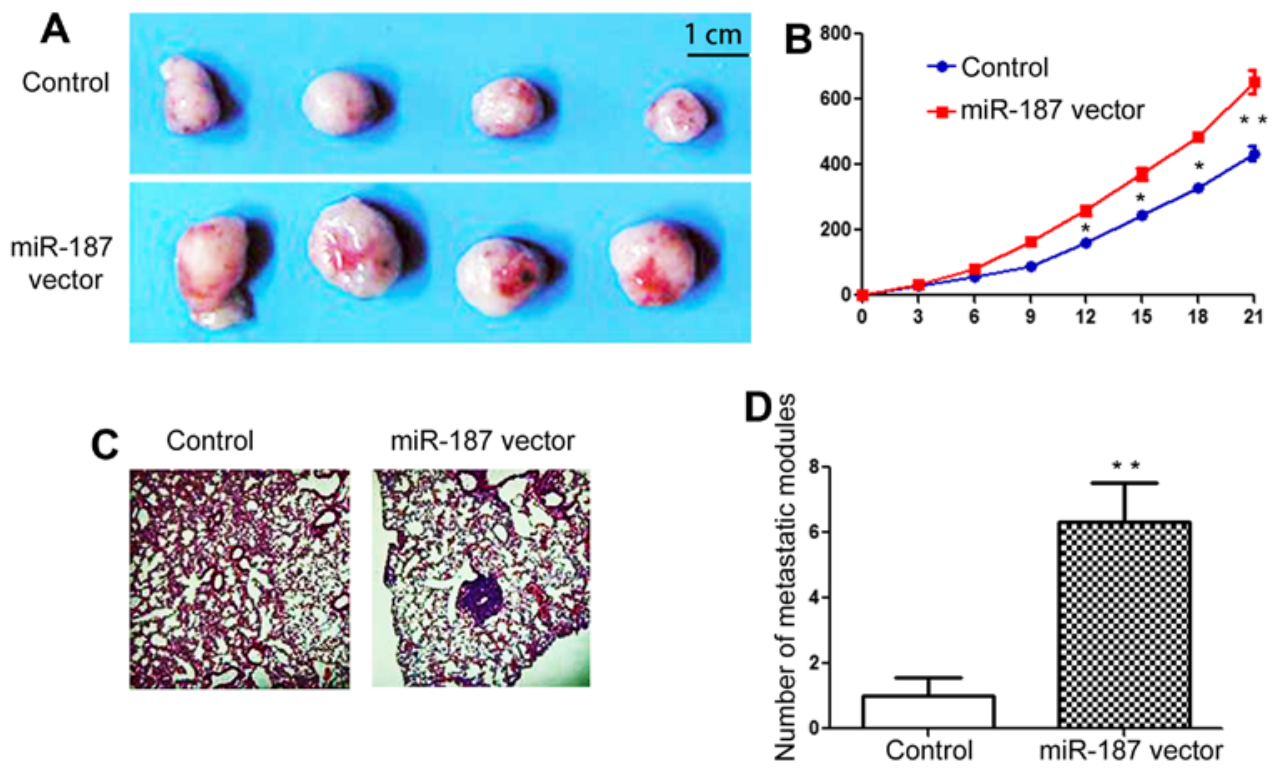

Figure 5. Overexpression of miR-187 promotes the growth and metastasis of SGC-7901 cells in vivo. (A) miR-187 overexpression significantly increased the in vivo growth of SGC-7901 cells in nude mice. (B) The growth curve of subcutaneous tumors between the control group and the miR-187 overexpression group. (C) Representative H\&E staining for lung metastasis between the control group and the miR-187 overexpression group. (D) miR-187 overexpression significantly increased the number of metastatic nodules in the lung of nude mice. ${ }^{*} \mathrm{P}<0.05,{ }^{* *} \mathrm{P}<0.01$.

\section{A}

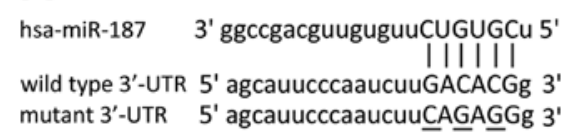

C
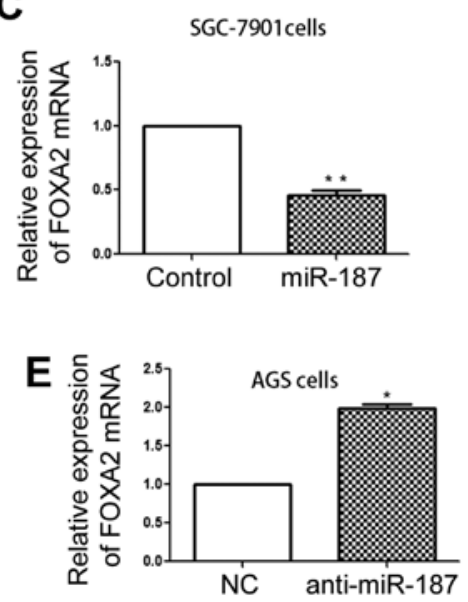

D
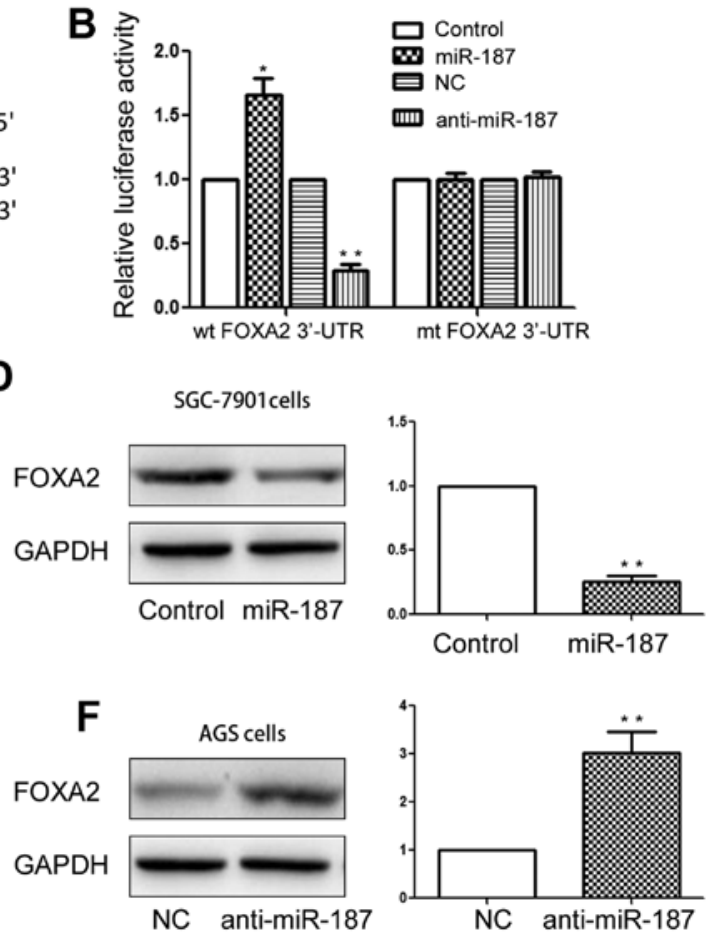

Figure 6. FOXA2 is a direct target of miR-187 in gastric cancer cells. (A) 3'-UTR of FOXA2 has the putative sequence for miR-187 binding. (B) miR-187 overexpression significantly suppressed while miR-187 inhibition significantly increased the luciferase activity of wild-type but not-mutant 3'-UTR of FOXA2. (C) miR-187 overexpression significantly reduced the mRNA level of FOXA2 in SGC-7901 cells. (D) miR-187 overexpression reduced the protein level of FOXA2 in SGC-7901 cells. (E) miR-187 knockdown increased FOXA2 mRNA level in AGS cells. (F) miR-187 knockdown increased FOXA2 protein level in AGS cells. ${ }^{*} \mathrm{P}<0.05,{ }^{* *} \mathrm{P}<0.01$.

after overexpression of miR-187 (Fig. 3C and D; $\mathrm{P}<0.05$ for wound healing assay, $\mathrm{P}<0.01$ for invasion assay). On the other hand, miR-187 inhibitor significantly decreased the expression of miR-187 in AGS cells (Fig. 4A; P<0.01). Subsequently, inhibition of miR-187 resulted in decreased cell proliferation (Fig. 4B; $\mathrm{P}<0.05$ ), migration (Fig. 4C; $\mathrm{P}<0.05$ ) and invasion
(Fig. 4D; P<0.01) of AGS cells. These data indicate that miR-187 promotes the proliferation, migration and invasion of GC cells in vitro.

miR-187 potentiates the growth and metastasis of GC cells in vivo. To further confirm the in vitro influence of miR-187 on 
A SGC-7901 cells overexpressing miR-187
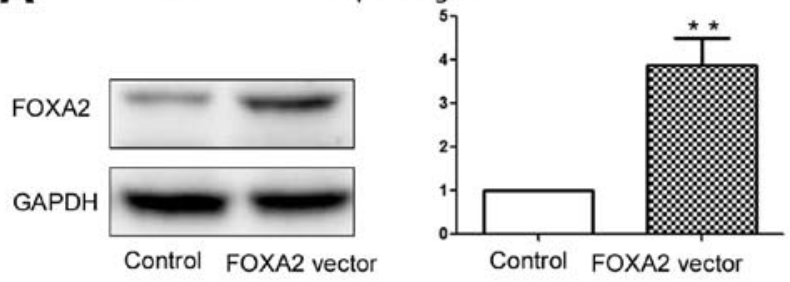

C

SGC-7901 cells overexpressing miR-187
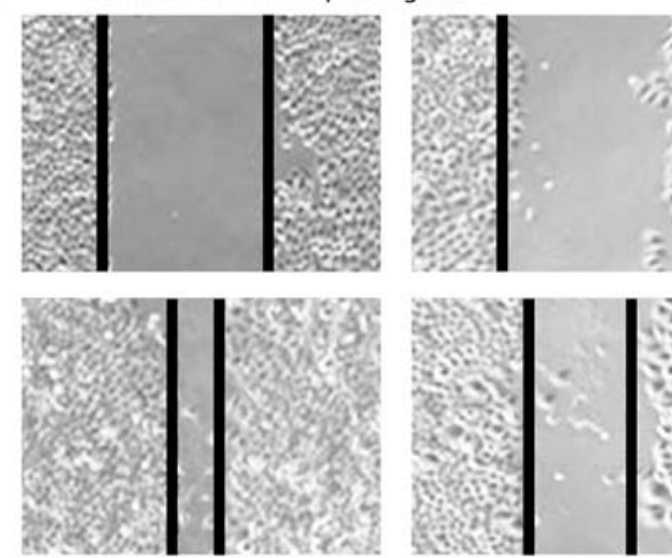

Control

D

SGC-7901 cells overexpressing miR-187

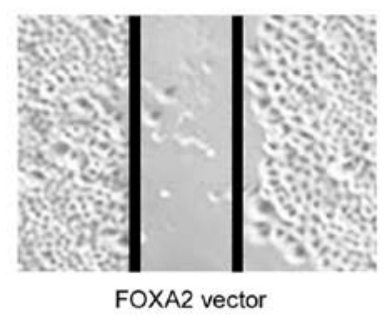

FOXA2 vector

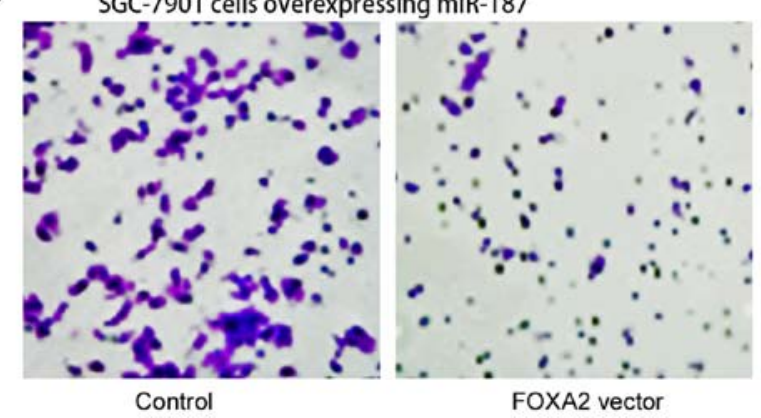

B SGC-7901 cells overexpressing miR-187

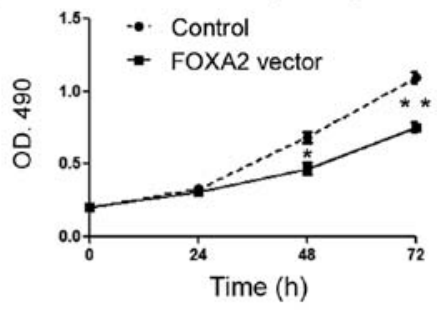

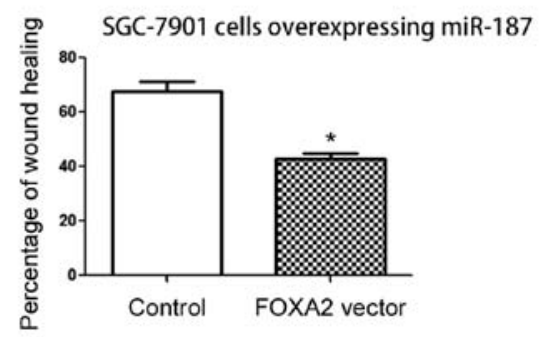

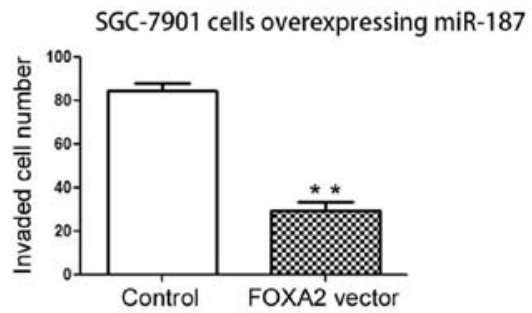

Figure 7. Overexpression of FOXA2 abrogates the promoting effects of miR-187 mimic on cell proliferation, migration and invasion. (A) FOXA2 expression vector significantly increased the expression of FOXA2 in SGC-7901 cells transfected with miR-187 mimic. Overexpression of FOXA2 abrogated the promoting effects of miR-187 overexpression on (B) cell proliferation, (C) migration, (D) invasion. ${ }^{*} \mathrm{P}<0.05,{ }^{* *} \mathrm{P}<0.01$.

GC cells, we further carried out nude mouse experiments to determine whether miR-187 could promote the in vivo growth and metastasis of GC cells. The result of subcutaneous tumor formation assay showed that the growth of SGC-7901 cells was significantly increased after overexpression of miR-187 (Fig. 5A and $\mathrm{B} ; \mathrm{P}<0.01)$. Moreover, the tail vein injection experiments in nude mice showed that overexpression of miR-187 significantly increased the lung metastasis of SGC-7901 cells (Fig. 5C and $\mathrm{D} ; \mathrm{P}<0.01)$. These data suggest that miR-187 potentiate the growth and metastasis of GC cells in vivo.

FOXA2 is a downstream target of miR-187 in GC cells. After confirming the functional influence of miR-187 in GC cells, we further investigated the underlying mechanism mediating the function of miR-187. The data from the database of TargetScan and miRNA showed that FOXA2 contained the potential binding sites for miR-187 (Fig. 6A), suggesting FOXA2 is a potential downstream target of miR-187. Then, we performed luciferase assay and found that overexpression of miR-187 significantly inhibited the luciferase activity of wild-type
3'-UTR of FOXA2 (Fig. 6B; P<0.01) while inhibiting miR-187 significantly increased the luciferase activity of wild-type FOXA2 3'-UTR (Fig. 6B; P<0.05). The influence of miR-187 overexpression and inhibition on the luciferase activity of wild-type 3'-UTR of FOXA2 was absent in the mutant 3'-UTR of FOXA2 (Fig. 6B). Furthermore, we performed qRT-PCR and western blot analysis to confirm that miR-187 could affect the expression of FOXA2 in GC cells. As suggested by Fig. 6C and $\mathrm{D}$, overexpression of miR-187 significantly decreased the mRNA $(\mathrm{P}<0.01)$ and protein $(\mathrm{P}<0.01)$ level of FOXA2 in SGC-7901 cells. On the other hand, inhibition of miR-187 significantly increased the expression of FOXA2 mRNA (Fig. 6E; $\mathrm{P}<0.05$ ) and protein (Fig. 6F; $\mathrm{P}<0.01$ ) in AGS cells.

FOXA2 mediates the biological functions of miR-187 in GC cells. Lastly, we examined whether FOXA2 could affect the biological function of miR-187 in gastric cancer cells. FOXA2 expression vector significantly increased the protein level of FOXA2 in SGC-7901 cells overexpressing miR-187 (Fig. 7A; $\mathrm{P}<0.01)$. Functionally, overexpression of FOXA2 abrogated 
A

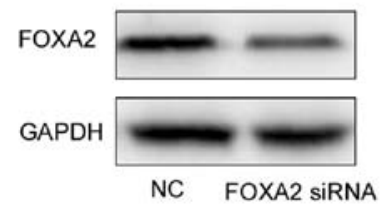

C

AGS cells with miR-187 knockdown

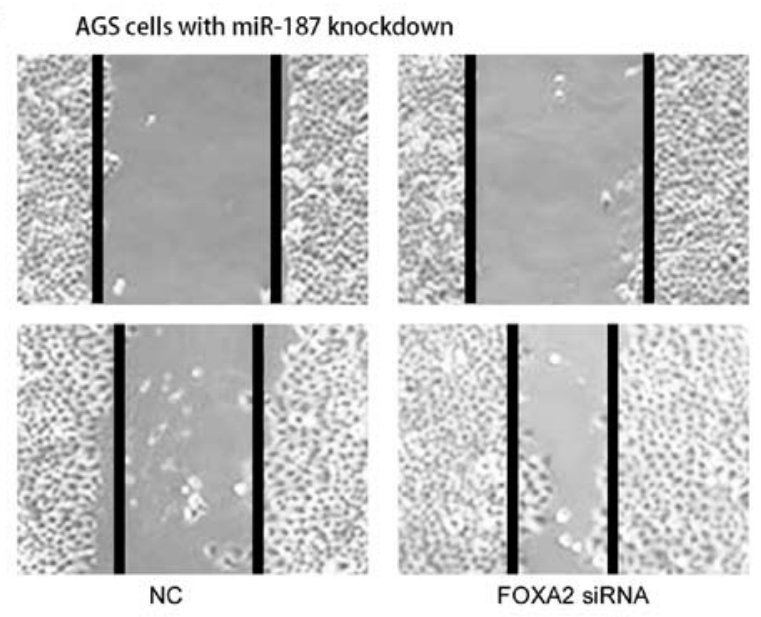

D
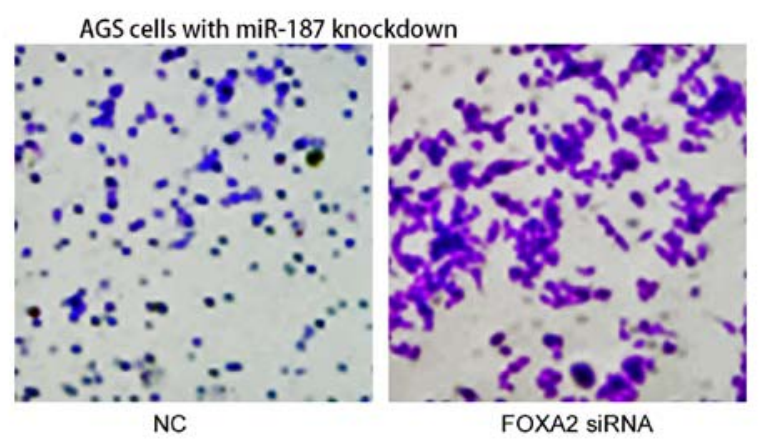

B

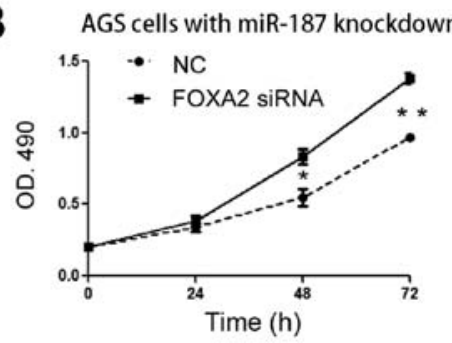

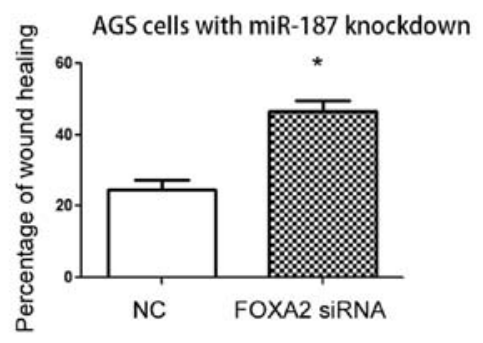

Figure 8. Inhibition of FOXA2 reverses the suppressive effects of miR-187 inhibitor on cell proliferation, migration and invasion. (A) FOXA2 siRNA significantly decreased FOXA2 expression in AGS cells transfected with miR-187 inhibitor. Knockdown of FOXA2 abrogated the suppressive effects of miR-187 inhibition on (B) cell proliferation, (C) migration, (D) invasion. ${ }^{*} \mathrm{P}<0.05,{ }^{* *} \mathrm{P}<0.01$.

the promoting effects of miR-187 mimic on the proliferation (Fig. 7B; $\mathrm{P}<0.05$ ), migration (Fig. 7C; $\mathrm{P}<0.05$ ) and invasion (Fig. 7D; P<0.01) of SGC-7901 cells. On the contrary, FOXA2 siRNA significantly decreased the expression of FOXA2 in AGS cells with miR-187 inhibition (Fig. 8A; $\mathrm{P}<0.05$ ). Inhibition of FOXA2 prevented the inhibitory effects of miR-187 inhibitor on the proliferation (Fig. 8B; $\mathrm{P}<0.01$ for MTT), migration (Fig. 8C; $\mathrm{P}<0.05$ ) and invasion (Fig. 8D; $\mathrm{P}<0.01)$ of AGS cells. These data indicate that FOXA2 is a functional mediator for miR-187 in GC cells.

\section{Discussion}

Biological functions of microRNAs in human diseases have been extensively investigated during the past two decades (15). In addition, increasing amount of studies showed that miRNAs were active players in the pathogenic process of human cancers (16). miRNAs have been found to be therapeutic targets and biomarkers for human cancers including GC $(17,18)$.

Among numerous miRNAs, miR-187 was recently found to be a novel cancer-associated microRNA. It could promote the development of non-small cell lung cancer by inhibiting Bcl-6 (11). The study involving breast cancer showed that miR-187 increased the invasive behavior of cancer cells (14). On the contrary, miR-187 expression was found to be decreased in the tissues of prostate cancer and was correlated with poor clinicopathological features of patients $(13,19)$. In colorectal cancer, miR-187 was found to prevent Smad-mediated epithelial-mesenchymal transition in colorectal cancer cells (12). Therefore, the expression and biological function of miR-187 in human cancers vary between different cancer types.

The present study found that miR-187 expression was significantly increased in GC tissues and cell lines. In addition, association analysis showed that miR-187 was associated with poor clinical features and poor prognosis of GC patients. The in vitro functional assays showed that overexpression of miR-187 could promote the proliferation and metastatic ability of SGC-7901 cells while inhibition of miR-187 decreased the proliferation and invasive ability of AGC cells. Furthermore, the in vivo experiments confirmed that miR-187 not only promoted the proliferation and invasive ability of GC cell in vitro, but also potentiated the growth and lung metastasis of 
GC in nude mice. Taken together, these data strongly demonstrated that miR-187 plays an oncogenic function in GC by promoting the growth and metastasis of GC cells.

After elucidating the biological function of miR-187 in GC, we further explored the underlying mechanisms mediating the function of miR-187 in GC. The data from public database showed that FOXA2 was a potential downstream target of miR-187. Interestingly, previous study showed that FOXA2 expression was decreased in GC tissues and could suppress the development and progression of GC both in vitro and in vivo $(20,21)$. Our luciferase assay data showed that altering miR-187 level could significantly affect the luciferase activity of wild-type 3'-UTR of FOXA2 while had no effect on that of mutant 3'-UTR of FOXA2. Moreover, overexpression of miR-187 significantly decreased while inhibition of miR-187 significantly increased the mRNA and protein expression of FOXA2 in GC cells. These data strongly showed that FOXA2 was a downstream target of miR-187 in GC cells. Lastly, we determined whether FOXA2 mediated the biological functions of miR-187 in GC. Our data showed that overexpression of FOXA2 abrogated the promoting effects of miR-187 mimic on the proliferation and metastasis of GC cells while knockdown of FOXA 2 reversed the inhibitory effects of miR-187 inhibitor on the proliferation and metastasis of GC cells. These data confirmed that FOXA2 was the functional mediator of miR-187 in GC cells. However, it is important that microRNAs usually have more than one downstream target in the cells. Therefore, it is possible that miR-187 has other downstream targets in gastric cancer cells which is worth investigation in the future.

In summary, we demonstrated for the first time that miR-187 was increased in GC tissues and cells. Increased expression level of miR-187 was associated with poor clinical features and prognosis of GC patients. Moreover, miR-187 was able to promote the growth and metastasis of GC cells. Mechanically, FOXA2 was confirmed to be the downstream target of miR-187 in GC and to mediate the functional influence of miR-187 on GC cells. Therefore, the present study indicates that miR-187 is potentially a biomarker and therapeutic target for GC patients.

\section{References}

1. Kamangar F, Dores GM and Anderson WF: Patterns of cancer incidence, mortality, and prevalence across five continents: Defining priorities to reduce cancer disparities in different geographic regions of the world. J Clin Oncol 24: 2137-2150, 2006.

2. Jemal A, Center MM, DeSantis C and Ward EM: Global patterns of cancer incidence and mortality rates and trends. Cancer Epidemiol Biomarkers Prev 19: 1893-1907, 2010.

3. Orditura M, Galizia G, Sforza V, Gambardella V, Fabozzi A, Laterza MM, Andreozzi F, Ventriglia J, Savastano B, Mabilia A, et al: Treatment of gastric cancer. World J Gastroenterol 20: 1635-1649, 2014.

4. Huang Y, Shen XJ, Zou Q, Wang SP, Tang SM and Zhang GZ Biological functions of microRNAs: A review. J Physiol Biochem 67: 129-139, 2011.
5. Iorio MV and Croce CM: microRNA involvement in human cancer. Carcinogenesis 33: 1126-1133, 2012.

6. Song S and Ajani JA: The role of microRNAs in cancers of the upper gastrointestinal tract. Nat Rev Gastroenterol Hepatol 10: 109-118, 2013.

7. Ohshima K, Inoue K, Fujiwara A, Hatakeyama K, Kanto K, Watanabe Y, Muramatsu K, Fukuda Y, Ogura S, Yamaguchi K, et al: Let-7 microRNA family is selectively secreted into the extracellular environment via exosomes in a metastatic gastric cancer cell line. PLoS One 5: e13247, 2010.

8. Peng S, Kuang Z, Sheng C, Zhang Y, Xu H and Cheng Q: Association of microRNA-196a-2 gene polymorphism with gastric cancer risk in a Chinese population. Dig Dis Sci 55: 2288-2293, 2010.

9. Wang HJ, Ruan HJ, He XJ, Ma YY, Jiang XT, Xia YJ, Ye ZY and Tao HQ: MicroRNA-101 is down-regulated in gastric cancer and involved in cell migration and invasion. Eur $\mathbf{J}$ Cancer 46: 2295-2303, 2010.

10. Lai KW, Koh KX, Loh M, Tada K, Subramaniam MM, Lim XY, Vaithilingam A, Salto-Tellez M, Iacopetta B, Ito Y, et al; Singapore Gastric Cancer Consortium: MicroRNA-130b regulates the tumour suppressor RUNX3 in gastric cancer. Eur J Cancer 46: 1456-1463, 2010.

11. Sun C, Li S, Yang C, Xi Y, Wang L, Zhang F and Li D: MicroRNA-187-3p mitigates non-small cell lung cancer (NSCLC) development through down-regulation of BCL6. Biochem Biophys Res Commun 471: 82-88, 2016.

12. Zhang F, Luo Y, Shao Z, Xu L, Liu X, Niu Y, Shi J, Sun X, Liu Y, Ding Y, et al: MicroRNA-187, a downstream effector of TGF $\beta$ pathway, suppresses Smad-mediated epithelial-mesenchymal transition in colorectal cancer. Cancer Lett 373: 203-213, 2016.

13. Casanova-Salas I, Masiá E, Armiñán A, Calatrava A, Mancarella C, Rubio-Briones J, Scotlandi K, Vicent MJ and López-Guerrero JA: MiR-187 targets the androgen-regulated gene ALDH1A3 in prostate cancer. PLoS One 10: e0125576, 2015.

14. Mulrane L, Madden SF, Brennan DJ, Gremel G, McGee SF, McNally S, Martin F, Crown JP, Jirström K, Higgins DG, et al: miR-187 is an independent prognostic factor in breast cancer and confers increased invasive potential in vitro. Clin Cancer Res 18: 6702-6713, 2012

15. Calin GA and Croce CM: MicroRNA signatures in human cancers. Nat Rev Cancer 6: 857-866, 2006.

16. Davis-Dusenbery BN and Hata A: MicroRNA in cancer: The Involvement of aberrant MicroRNA biogenesis regulatory pathways. Genes Cancer 1: 1100-1114, 2010.

17. Liu R, Zhang C, Hu Z, Li G, Wang C, Yang C, Huang D, Chen X, Zhang $\mathrm{H}$, Zhuang R, et al: A five-microRNA signature identified from genome-wide serum microRNA expression profiling serves as a fingerprint for gastric cancer diagnosis. Eur J Cancer 47: 784-791, 2011.

18. Slack FJ and Weidhaas JB: MicroRNA in cancer prognosis. N Engl J Med 359: 2720-2722, 2008

19. Casanova-Salas I, Rubio-Briones J, Calatrava A, Mancarella C, Masiá E, Casanova J, Fernández-Serra A, Rubio L, RamírezBackhaus M, Armiñán A, et al: Identification of miR-187 and miR-182 as biomarkers of early diagnosis and prognosis in patients with prostate cancer treated with radical prostatectomy. J Urol 192: 252-259, 2014

20. Katoh M and Katoh M: Integrative genomic analyses of CXCR4: Transcriptional regulation of CXCR4 based on TGFbeta, Nodal, Activin signaling and POU5F1, FOXA2, FOXC2, FOXH1, SOX17, and GFI1 transcription factors. Int J Oncol 36: 415-420, 2010.

21. Zhu C-P, Wang J, Shi B, Hu PF, Ning BF, Zhang Q, Chen F, Chen WS, Zhang $X$ and Xie WF: The transcription factor FOXA2 suppresses gastric tumorigenesis in vitro and in vivo. Dig Dis Sci 60: 109-117, 2015. 